\title{
Hypo-elasticity and Plasticity. II
}

\author{
A. E. GREEN
}

1. Introduction. In a previous paper by the present writer (1956) TRUESDELL's theory of hypo-elasticity (1955) was used to derive equations suitable for plastic flow of an incompressible work-hardening body. The present paper is concerned with the corresponding results for compressible bodies. In addition, the plastic flow of compressible bodies with zero work-hardening is discussed. In this case, by considering a simple special form of the general equations in the non-plastic region, we show that the yield criterion must then be of the von Mises type. Among the possible corresponding equations of plastic flow we find equations which are usually used in the theory of plasticity (Hill 1950, Prager \& Hodge 1951), except that "rate of change of stress" is defined here to have the correct tensorial form. Work which is related to but different from that of the present paper has been published recently by Thomas (1955).

2. Notation. We use a fixed system of general curvilinear coordinates $x^{\boldsymbol{i}}$ with corresponding metric tensors $g_{r s}(x), g^{r s}(x)$. If $v_{i}$ is the covariant velocity vector the rate of deformation tensor $d_{i j}$ is defined by

$$
d_{i j}=\frac{1}{2}\left(v_{i, j}+v_{i, i}\right)
$$

where a comma denotes covariant differentiation. We denote the stress tensor by $2 \mu s_{i j}$ where $\mu$ is one of the usual Lamé constants of classical elasticity. The stress deviator and the rate of strain deviator, denoted respectively by $2 \mu t_{i i}$ and $f_{i j}$, are defined by the equations

$$
t_{i}^{i}=s_{i}^{i}-\frac{1}{3} s_{r}^{r} \delta_{i}^{i}, \quad f_{i}^{i}=d_{i}^{i}-\frac{1}{3} d_{r}^{r} \delta_{i}^{i}
$$

and we see that

$$
t_{i}^{i}=0, \quad f_{i}^{i}=0 .
$$

In formulating constitutive equations of the type

$$
\text { rate of stress }=f \text { (stress, rate of deformation), }
$$


Truesdell (1955) devised a procedure for obtaining a definition of "rate of stress" which has the correct tensorial character. Alternative procedures were employed by Thomas (1955a,c) and Green (1956), the latter using a method due to OLdRoyd (1950). Here we quote the definition of "rate of stress" from Oldroyd (1950) and Green (1956) as

$$
\tilde{s}^{i k}=\frac{\partial s^{i k}}{\partial t}+v^{m} s_{, m}^{i k}-v_{, m}^{i} s^{m k}-v_{, m}^{i} s^{i m}
$$

In mixed form this may be written

$$
g_{j k} \tilde{s}^{i k}=\frac{\sim}{g_{j k} s^{i k}}-\tilde{g}_{j k} s^{i k}=\tilde{s}_{j}^{i}-2 s_{k}^{i} d_{j}^{k},
$$

where

$$
\begin{aligned}
\tilde{s}_{j}^{i} & =\frac{\partial s_{j}^{i}}{\partial t}+v^{m} s_{j, m}^{i}+v_{, i}^{m} s_{m}^{i}-v_{, m}^{i} s_{i}^{m} \\
& =\frac{\partial s_{i}^{i}}{\partial t}+v^{m} \frac{\partial s_{j}^{i}}{\partial x^{m}}+\frac{\partial v^{m}}{\partial x^{j}} s_{m}^{i}-\frac{\partial v^{i}}{\partial x^{m}} s_{i}^{m}
\end{aligned}
$$

If $\rho$ is density and body forces are zero the equations of motion and continuity are

$$
\begin{gathered}
2 \mu s_{, i}^{i j}=\rho\left(\frac{\partial v^{i}}{\partial t}+v_{, j}^{i} v^{j}\right), \\
\frac{\partial \rho}{\partial t}+\left(\rho v^{i}\right)_{, i}=0 .
\end{gathered}
$$

Apart from a factor $2 \mu$ the stress power is

$$
\Phi=s_{j}^{i} d_{i}^{j}=t_{j}^{i} f_{i}^{j}+\frac{1}{3} s_{m}^{m} d_{n}^{n} .
$$

3. Isotropic hypo-elastic bodies. TRUEsDell (1955) has obtained the most general analytic isotropic relation of the form (2.4) in which no modulus of the material of dimension of time is present, and calls such a relation the constitutive equations of an isotropic hypo-elastic body. Here we use (2.6) and write TRUESDELL's equations in the slightly different but equivalent form

$$
\begin{aligned}
\tilde{s}_{i}^{i}-2 s_{k}^{i} d_{i}^{k}=g_{0} d_{m}^{m} \delta_{j}^{i}+g_{1} d_{i}^{i}+ & g_{2} d_{m}^{m} s_{i}^{i}+g_{3} \Phi \delta_{i}^{i} \\
& +\frac{1}{2} g_{4}\left(d_{k}^{i} s_{i}^{k}+s_{k}^{i} d_{i}^{k}\right)+g_{5} d_{m}^{m} s_{k}^{i} s_{i}^{k} \\
& +g_{0} \Phi s_{i}^{i}+g_{7} \Psi \delta_{i}^{i}+\frac{1}{2} g_{8}\left(d_{m}^{i} s_{n}^{m} s_{i}^{n}+s_{m}^{i} s_{n}^{m} d_{i}^{n}\right) \\
& +g_{9} \Phi s_{k}^{i} s_{i}^{k}+g_{10} \Psi s_{i}^{i}+g_{11} \Psi s_{k}^{i} s_{i}^{k} \\
\Phi= & s_{j}^{i} d_{i}^{i}, \quad \Psi=s_{j}^{i} s_{k}^{i} d_{i}^{k},
\end{aligned}
$$


where $g_{0}, g_{1}, \cdots, g_{11}$ are dimensionless analytic functions of the three principal invariants

$$
s_{i}^{i}, \quad \frac{1}{2} s_{j}^{i} s_{i}^{j}, \quad \frac{1}{3} s_{j}^{i} s_{k}^{j} s_{i}^{k},
$$

of $s_{j}^{i}$.

Using (2.2) and (2.3) equations (3.1) may be written in the form

$$
\begin{aligned}
\tilde{t}_{j}^{i}+\frac{1}{3} \tilde{s}_{m}^{m} \delta_{j}^{i}-2 t_{k}^{i} f_{i}^{k}=\left(h_{0} \delta_{j}^{i}+\right. & \left.h_{2} t_{j}^{i}+h_{5} t_{k}^{i} t_{j}^{k}\right) d_{m}^{m}+h_{1} f_{j}^{i} \\
& +\frac{1}{2} h_{4}\left(f_{k}^{i} t_{j}^{k}+t_{k}^{i} f_{j}^{k}\right)+\left(h_{3} M+h_{7} N\right) \delta_{i}^{i} \\
& +\left(h_{6} M+h_{10} N\right) t_{j}^{i}+\left(h_{9} M+h_{11} N\right) t_{k}^{i} t_{i}^{k} \\
& +\frac{1}{2} h_{8}\left(f_{m}^{i} t_{n}^{m} t_{j}^{n}+t_{m}^{i} t_{n}^{m} f_{j}^{n}\right),
\end{aligned}
$$

where

$$
M=t_{i}^{i} f_{i}^{i}, \quad N=t_{j}^{i} t_{k}^{i} f_{i}^{k},
$$

and where $h_{0}, h_{1}, \cdots, h_{11}$ are dimensionless analytic functions of the invariants

$$
s_{i}^{i}, \quad J=\frac{1}{2} t_{j}^{i} t_{i}^{j}, \quad K=\frac{1}{3} t_{j}^{i} t_{k}^{i} t_{i}^{k} .
$$

If we contract equations (3.4) and use (2.3) and (3.5) we obtain

$$
\begin{aligned}
\tilde{s}_{m}^{m}=\left(3 h_{0}+2 h_{5} J\right) d_{m}^{m} & +\left(h_{4}+2+3 h_{3}+2 h_{9} J\right) M \\
& +\left(3 h_{7}+h_{8}+2 h_{11} J\right) N .
\end{aligned}
$$

Also

$$
\Phi=M+\frac{1}{3} s_{m}^{m} d_{n}^{n}, \quad \Psi=N+\frac{2}{3} M s_{m}^{m}+\frac{2}{3} J d_{m}^{m}+\frac{1}{9} d_{i}^{i} s_{m}^{m} s_{n}^{n} .
$$

In this paper we wish to use the above theory of hypo-elasticity in order to obtain equations which are suitable for problems of plastic flow of metals. We therefore consider a special case of (3.4) which is such that $\tilde{t}_{i}^{i}$ is independent of the stress invariant $s_{m}^{m}$ and the rate of deformation invariant $d_{n}^{n}$. This condition can be satisfied if

$$
h_{2} \equiv h_{5} \equiv 0, \quad \tilde{s}_{m}^{m}=3 h_{0} d_{m}^{m},
$$

and if $h_{0}, h_{1}, \cdots, h_{11}$ are functions only of $J$ and $K$. In this case (3.4) reduces to $\tilde{t}_{i}^{i}=h_{1} f_{j}^{i}+2 t_{k}^{i} f_{j}^{k}+\frac{1}{2} h_{4}\left(f_{k}^{i} t_{j}^{k}+t_{k}^{i} f_{i}^{k}\right)$

$$
\begin{aligned}
& +\left(h_{3} M+h_{7} N\right) \delta_{i}^{i}+\left(h_{6} M+h_{10} N\right) t_{i}^{i} \\
& +\left(h_{9} M+h_{11} N\right) t_{k}^{i} t_{j}^{k}+\frac{1}{2} h_{8}\left(f_{m}^{i} t_{n}^{m} t_{j}^{n}+t_{m}^{i} t_{n}^{m} f_{i}^{n}\right) .
\end{aligned}
$$

Also, if we use (3.9) in (3.7) and require the resulting equation to hold for all non-zero values of $M$ and $N$ we have

$$
\begin{aligned}
h_{4}+2+3 h_{3}+2 h_{9} J & =0, \\
3 h_{7}+h_{8}+2 h_{11} J & =0 .
\end{aligned}
$$


These results are also obtained directly by contracting equations (3.10).

From (3.10) we obtain two further results which will be used below. If we multiply (3.10) by $t_{i}^{i}$ and use the identity

$$
t_{m}^{i} t_{n}^{m} t_{j}^{n}=K \delta_{j}^{i}+J t_{j}^{i}
$$

we have

$\tilde{J}=\left[h_{1}+\left(2 h_{6}+h_{8}\right) J+3 h_{9} K\right] M+\left[h_{4}+2+2 h_{10} J+3 h_{11} K\right] N$.

Similarly, multiplying (3.10) by $t_{k}^{i} t_{i}^{k}$, and using (3.11), we have

$$
\tilde{K}=\left[-h_{3} J+\left(3 h_{6}+h_{8}\right) K\right] M+\left[h_{1}-h_{7} J+3 h_{10} K\right] N .
$$

4. Loading and unloading. From (3.8) we see that the stress power $\Phi$ is

$$
\Phi=M+\frac{1}{3} s_{m}^{m} d_{n}^{n}
$$

so that $M=t_{i}^{i} f_{i}^{i}$ represents the stress power* due to distortion of elements (as opposed to change of volume) and vanishes when the stress $s_{i}^{i}=p \delta_{i}^{i}$ or when $d_{i}^{i}=d \delta_{i}^{i}$, where $p$ and $d$ are scalars. $\dagger$ We assume that during a single loading the constitutive equations are given by (3.9) and (3.10), subject to (3.11), and to the condition $M>0$.

For a single unloading we assume constitutive equations of a similar general type, i.e.

$$
\begin{gathered}
\tilde{s}_{m}^{m}=3 h_{0}^{\prime} d_{m}^{m} \\
\tilde{t}_{i}^{i}=h_{1}^{\prime} f_{i}^{i}+2 t_{k}^{i} f_{i}^{k}+\frac{1}{2} h_{4}^{\prime}\left(f_{k}^{i} t_{i}^{k}+t_{k}^{i} f_{i}^{k}\right) \\
+\left(h_{3}^{\prime} M+h_{7}^{\prime} N\right) \delta_{i}^{i}+\left(h_{6}^{\prime} M+h_{10}^{\prime} N\right) t_{i}^{i} \\
+\left(h_{9}^{\prime} M+h_{11}^{\prime} N\right) t_{k}^{i} t_{j}^{k}+\frac{1}{2} h_{8}^{\prime}\left(f_{m}^{i} t_{n}^{m} t_{i}^{n}+t_{m}^{i} t_{n}^{m} f_{i}^{n}\right)
\end{gathered}
$$

where

$$
\begin{aligned}
h_{4}^{\prime}+2+3 h_{3}^{\prime}+2 h_{9}^{\prime} J & =0 \\
3 h_{7}^{\prime}+h_{8}^{\prime}+2 h_{11}^{\prime} J & =0
\end{aligned}
$$

and where $h_{1}^{\prime}, h_{2}^{\prime}, \cdots, h_{11}^{\prime}$ are new dimensionless analytic functions of the invariants $J, K$. Equations (4.1), (4.2) are valid for $M<0$. In addition, we regard the constitutive equations (3.9), (3.10) for loading and (4.1), (4.2) for unloading as suitable for plastic flow of metals only if the total dissipation of energy, per unit mass, in any complete loading and unloading to the same state of stress, is non-negative. We do not attempt here to express this condition

$*_{i . e .,}$ rate at which work is done per unit time per unit volume of the present configuration. $\dagger M$ may, of course, vanish in other cases. 
in a general mathematical form but it must be kept in mind when specific loading and unloading equations are used.

We now impose the condition that equations (3.9) and (3.10) should be identical with equations (4.1) and (4.2) respectively when $M=0$. This condition can be satisfied if

$$
\begin{gathered}
h_{0}^{\prime} \equiv h_{0}, \quad h_{1}^{\prime} \equiv h_{1}, \quad h_{4}^{\prime} \equiv h_{4}, \quad h_{7}^{\prime} \equiv h_{7}, \\
h_{8}^{\prime} \equiv h_{8}, \quad h_{10}^{\prime} \equiv h_{10}, \quad h_{11}^{\prime} \equiv h_{11} .
\end{gathered}
$$

The above discussion has been restricted to a single loading followed by a complete unloading. For further loading or unloading we could use the same sets of equations, one for $M>0$ and the other for $M<0$, subject to the restriction stated about total work of the deviatoric stresses. Alternatively we could introduce entirely new constitutive equations of the same general forms for each successive loading or unloading, subject to continuity of the forms when $M=0$. We will not, however, pursue this discussion further here.

5. Special constitutive equations. Truesdell (1955) has shown that the equations of the theory of hypo-elasticity reduce to those of the classical linear theory of elasticity under the assumptions usual in formulating that theory. In order to include the classical linear theory of elasticity as a first approximation in loading or unloading we take

$$
h_{0}=h_{0}^{\prime}=\frac{1+\nu}{3(1-2 \nu)}, \quad h_{1}=h_{1}^{\prime}=1,
$$

when $J=K=0$, where $\nu$ is the usual Poisson ratio.

Remembering (5.1) we now consider the simplest set of constitutive equations (4.1) and (4.2) for unloading, given by

$$
\begin{gathered}
h_{0}^{\prime}=\frac{1+\nu}{3(1-2 \nu)}, \quad h_{1}^{\prime}=1, \\
h_{3}^{\prime}=h_{6}^{\prime}=h_{7}^{\prime}=h_{8}^{\prime}=h_{9}^{\prime}=h_{10}^{\prime}=h_{11}^{\prime}=0,
\end{gathered}
$$

for all values of $J, K$. In view of (4.3) we then have

$$
h_{4}^{\prime}=-2 .
$$

With (5.2) and (5.3), equations (4.1) and (4.2) become

$$
\begin{gathered}
\tilde{s}_{m}^{m}=\frac{1+\nu}{1-2 \nu} d_{m}^{m}, \\
\tilde{t}_{i}^{i}+f_{k}^{i} t_{i}^{k}-t_{k}^{i} f_{j}^{k}=f_{i}^{i},
\end{gathered}
$$

subject to $M \leqq 0$. 
NoLL (1955) has shown that every isotropic elastic body is also an isotropic hypo-elastic body, where "elastic" is used in the Cauchy sense. Although equations (5.4) reduce to those of the classical linear theory of elasticity under the usual assumptions it is not necessarily true that (5.4) are elastic equations in general. In the case of simple extension, however, the equations yield a linear relation between stress and strain provided that strain is interpreted as logarithmic strain.

From (4.4), (5.2), (5.3) and (3.11), we have

$$
\begin{gathered}
h_{0} \equiv \frac{1+\nu}{3(1-2 \nu)}, \quad h_{1} \equiv 1, \quad h_{4} \equiv-2, \quad 3 h_{3}+2 h_{9} J \equiv 0, \\
h_{7} \equiv h_{8} \equiv h_{10} \equiv h_{11} \equiv 0 .
\end{gathered}
$$

The corresponding equations for loading are therefore

$$
\begin{gathered}
\tilde{s}_{m}^{m}=\frac{1+\nu}{1-2 \nu} d_{m}^{m}, \\
t_{i}^{i}+f_{k}^{i} t_{i}^{k}-t_{k}^{i} f_{i}^{k}=f_{i}^{i}+\left(-\frac{2}{3} h_{9} J \delta_{i}^{i}+h_{6} t_{i}^{i}+h_{9} t_{k}^{i} t_{j}^{k}\right) M,
\end{gathered}
$$

provided $M \geqq 0$.

The condition about total dissipation of energy mentioned in $\$ 4$ may impose restrictions on the functions $h_{6}, h_{9}$, but we do not discuss these here. A special case of (5.6), of some interest, is given by

$$
h_{6} \equiv-\frac{2 \alpha^{2}}{3}, \quad h_{9} \equiv 0,
$$

where $\alpha$ is a constant. Using (5.7) the second equation in (5.6) becomes

$$
t_{i}^{i}+f_{k}^{i} t_{i}^{k}-t_{k}^{i} f_{i}^{k}=f_{i}^{i}-\frac{2}{3} \alpha^{2} M t_{i}^{i} \quad(M \geqq 0) .
$$

Also, from (3.13),

$$
\tilde{J}=\left(1-\frac{4 \alpha^{2}}{3} J\right) M
$$

which suggests a "yield" condition of the von Mises type. Truesdell (1956) has pointed out that "yield" in this sense may never occur, and even if it does occur at infinite strain "hypo-elastic yield" may occur previously at finite strain. For simple shear TRUESDELL has shown that von Mises yield only occurs when $\alpha$ is large, but that previously the shear stress attains a maximum value as a function of shear strain, finally sinking to the von Mises yield value asymptotically. The corresponding equation to (5.8) was studied for an incompressible body by Green (1956). In this case $d_{i}^{i}=f_{i}^{i}$ and the first equation in (5.6) is omitted, $s_{m}^{m}$ being replaced by an arbitrary hydrostatic pressure. Green (1956) studied the case of simple shear but only integrated the equations approximately for 
large $\alpha$, thus overlooking the interesting result mentioned above, since when $\alpha \rightarrow \infty$ the von Mises and hypo-elastic yield points coincide and are reached asymptotically. GReEN's results are valid when terms of $O\left(1 / \alpha^{3}\right)$ are neglected in the final expressions for the stresses. The same situation may also occur in the torsion problem considered by Green (1956).

6. Plastic flow with zero work-hardening. Here we take constitutive equations for "plastic" flow to be (3.9) and (3.10), subject to conditions (3.11) and to the restriction $M>0$. We now add a condition, implied by the idea of zero "workhardening", in the form

$$
F(J, K)=c,
$$

where $F$ is a function of the invariants $J, K$ of the stress deviator, and $c$ is a positive constant. Hence

$$
\frac{\partial F}{\partial J} \tilde{J}+\frac{\partial F}{\partial K} \tilde{K}=0
$$

If we substitute $\tilde{J}, \tilde{K}$ from (3.13) and (3.14) into (6.2) and require the resulting equation to hold for all non-zero values of $M$ and $N$ we find that

$$
\begin{array}{r}
{\left[h_{1}+\left(2 h_{6}+h_{8}\right) J+3 h_{9} K\right] \frac{\partial F}{\partial J}+\left[-h_{3} J+\left(3 h_{6}+h_{8}\right) K\right] \frac{\partial F}{\partial K}=0} \\
{\left[h_{4}+2+2 h_{10} J+3 h_{11} K\right] \frac{\partial F}{\partial J}+\left[h_{1}-h_{7} J+3 h_{10} K\right] \frac{\partial F}{\partial K}=0 .}
\end{array}
$$

In the "non-plastic" range* we assume that

$$
F(J, K)<c
$$

and that the constitutive equations are given by (4.1) and (4.2), subject to conditions (4.3). In this range loading corresponds to $M>0$ and unloading to $M<0$. We add the condition that in the transition stage when

$$
F(J, K)=c, \quad M=0,
$$

the equations in the "plastic" and "non-plastic" regions are identical, which leads to equations (4.4).

As in $\$ 4$ we accept constitutive equations as suitable for the plastic flow of metals if the total dissipation of energy, per unit mass, in any complete loading (in the plastic or non-plastic regions) and unloading to the same state of stress, is non-negative.

\footnotetext{
*We use the term "non-plastic" since the equations at this stage are not necessarily those for an elastic body.
} 
7. Special constitutive equations when work-hardening is zero. Following an argument similar to that used in $\$ 5$ we adopt equations (5.4), namely,

$$
\begin{gathered}
\tilde{s}_{m}^{m}=\frac{1+\nu}{1-2 \nu} d_{m}^{m}, \\
\tilde{t}_{i}^{i}+f_{k}^{i} t_{i}^{k}-t_{k}^{i} f_{j}^{k}=f_{i}^{i},
\end{gathered}
$$

in the non-plastic region for which

$$
F(J, K)<c .
$$

Here, however, $M$ is not restricted in sign. It again follows that the corresponding equations for the plastic region are

$$
\begin{gathered}
\tilde{s}_{m}^{m}=\frac{1+\nu}{1-2 \nu} d_{m}^{m}, \\
\tilde{t}_{j}^{i}+f_{k}^{i} t_{j}^{k}-t_{k}^{i} f_{j}^{k}=f_{j}^{i}+\left(-\frac{2}{3} h_{9} J \delta_{j}^{i}+h_{6} t_{j}^{i}+h_{9} t_{k}^{i} t_{j}^{k}\right) M
\end{gathered}
$$

together with the yield condition

$$
F(J, K)=c .
$$

Also, using (5.5), equations (6.3) reduce to

$$
\begin{gathered}
\left(1+2 h_{6} J+3 h_{9} K\right) \frac{\partial F}{\partial J}+\left(-h_{3} J+3 h_{6} K\right) \frac{\partial F}{\partial K}=0, \\
\frac{\partial F}{\partial K}=0 .
\end{gathered}
$$

Hence $F$ is independent of $K$ and, without loss of generality, equation (7.4) can be replaced by

$$
F \equiv J=c,
$$

which is the well-known yield criterion of von Mises. The first equation in (7.5) then becomes

$$
1+2 h_{6} J+3 h_{9} K=0 .
$$

The condition that the total dissipation of energy per unit mass in a complete stress cycle is non-negative, probably imposes further restrictions on $h_{6}, h_{9}$ but it appears to be difficult to discuss this in a general manner. Instead, we restrict attention to the case when $h_{6}, h_{9}$ are independent of $K$ so that, since (7.7) must be true for all values of $K$,

$$
h_{9} \equiv 0, \quad h_{6} \equiv-\frac{1}{2 J} \equiv-\frac{1}{2 c} .
$$


With these values equations (7.3) become

$$
\begin{gathered}
\tilde{s}_{m}^{m}=\frac{1+\nu}{1-2 \nu} d_{m}^{m}, \\
\tilde{t}_{j}^{i}+f_{k}^{i} t_{j}^{k}-t_{k}^{i} f_{j}^{k}=f_{j}^{i}-\frac{M}{2 c} t_{j}^{i} \quad(M>0) .
\end{gathered}
$$

These equations are used with the yield criterion $J=c$.

Equations (7.1) and (7.9) are the usual Prandtl-Reuss equations for the nonplastic and plastic regions in a body, when work-hardening is zero, except that $\tilde{t}_{j}^{i}+f_{k}^{i} t_{j}^{k}-t_{k}^{i} f_{j}^{k}$ replaces the partial derivative of stress with respect to time (Prager \& Hodge 1951), or the total derivative with respect to time (HILL 1950). We observe from (2.7) that

$$
\tilde{s}_{m}^{m}=\frac{\partial s_{m}^{m}}{\partial t}+v^{r} \frac{\partial s_{m}^{m}}{\partial x^{r}}
$$

which is the total time derivative. A further investigation is needed in order to decide when we may approximate to $\tilde{t}_{i}^{i}+f_{k}^{i} t_{j}^{k}-t_{k}^{i} f_{j}^{k}$ by partial or total time derivatives.

We note that when expressed in rectangular cartesian coordinates $\tilde{t}_{j}^{i}+f_{k}^{i} t_{j}^{k}-$ $t_{k}^{i} f_{i}^{k}$, which is equal to $\tilde{t}_{i}^{i}+d_{k}^{i} t_{j}^{k}-t_{k}^{i} d_{i}^{k}$, is the same as the expression derived by Thomas $(1955 \mathrm{a}, \mathrm{c})$ for "rate of stress", if we replace stress by deviatoric stress.

8. Note on rigid-plastic equations (added 16 January 1956). Related work by Thomas (1955b) was mentioned in $\$ 1$. The writer has now seen a further paper by Thомаs (1955d) in which he considers incompressible plastic flow of the von Mises type. It appears to be worth observing that the usual von Mises equations for rigid-plastic flow can be obtained from the equations of $\$ 7$ by a limiting process. These equations are in non-dimensional form. If we restore the factor $2 \mu$ and use $s_{i}^{i}, t_{i}^{i}$ for the ordinary stress and deviatoric stress tensors we see that equations (7.9) for plastic flow become

$$
\begin{gathered}
\frac{\tilde{s}_{m}^{m}}{2 \mu}=\frac{1+\nu}{1-2 \nu} d_{m}^{m}, \\
\left(\tilde{t}_{i}^{i}+f_{k}^{i} t_{j}^{k}-t_{k}^{i} f_{j}^{k}\right) /(2 \mu)=f_{j}^{i}-\frac{M}{2 k^{2}} t_{j}^{i} \quad(M>0),
\end{gathered}
$$

where now

$$
M=f_{i}^{i} t_{i}^{i}, \quad J=\frac{1}{2} t_{j}^{i} t_{i}^{j}=k^{2}, \quad k^{2}=4 \mu^{2} c .
$$

For non-plastic flow

$$
\left(\tilde{t}_{j}^{i}+f_{k}^{i} t_{j}^{k}-t_{k}^{i} f_{j}^{k}\right) /(2 \mu)=f_{j}^{i} \quad\left(J<k^{2}\right),
$$

together with (8.1). 
We now assume that $\mu \rightarrow \infty, c \rightarrow 0$ in such a way that $k^{2}$ and the stress tensor remain fixed. From (8.1) and (8.4) we see that, in the limit, for non-plastic flow

$$
d_{m}^{m}=0, \quad f_{i}^{i}=0,
$$

so that the body is rigid when $J<k^{2}$. On the other hand, during plastic flow when $J=k^{2}$, we see from (8.1) and (8.2) that, in the limit,

$$
d_{m}^{m}=0, \quad t_{k}^{i}=\frac{2 k^{2}}{M} f_{k}^{i}, \quad M=f_{j}^{i} t_{i}^{i}>0 .
$$

Plastic flow is now incompressible and $s_{m}^{m}$ becomes an arbitrary hydrostatic pressure. Also, from (8.6),

$$
M^{2}=2 k^{2} f_{i}^{i} f_{i}^{i} .
$$

Equations (8.6) are the usual von Mises equations for rigid-plastic flow.

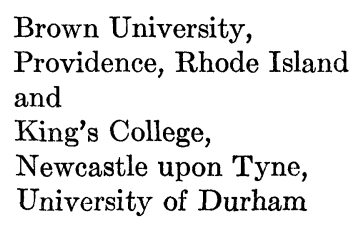

The results presented in this paper were obtained in the course of research sponsored by the Office of Ordnance Research, Department of the Army, under Contract DA-19-020-ORD-3487.

\section{REFERENCES}

Green, A. E. (1956), Proc. Roy. Soc. A234, 46-59.

Hrus, R. (1950), The Mathematical Theory of Plasticity, Oxford.

Noul, W. (1955), J. Rational Mech. Anal., 4, 3.

Oldroyd, J. G. (1950), Proc. Roy. Soc., A200, 523.

Prager, W. \& Hodge, P. G. (1950), Theory of Perfectly Plastic Solids, New York.

Thomas, T. Y. (1955a), Proc. N. Acad. Sci., 41, 716.

Thomas, T. Y. (1955b), Proc. N. Acad. Soc., 41, 720.

Thomas, T. Y. (1955c), Proc. N. Acad. Sci., 41, 762.

Thomas, T. Y. (1955d), Proc. N. Acad. Sci., 41, 908.

Truespell, C. (1955), J. Rational Mech. Anal., 4, 83.

Truesdell, C. (1956), J. Applied Phys., to appear. 